UDC 517.54

A. SAIRAm KaliRAJ

\title{
INJECTIVITY OF SECTIONS OF CLOSE-TO-CONVEX HARMONIC MAPPINGS WITH FUNCTIONS CONVEX IN ONE DIRECTION AS ANALYTIC PART
}

\begin{abstract}
In this article, we prove a two-points distortion theorem and obtain sharp coefficient estimates for the families of close-toconvex harmonic mappings whose analytic part is a function convex in one direction. By making use of these results, we determine the radius of univalence of sections of these families in terms of zeros of a certain equation. the lower bound for the radius of univalence has been obtained explicitly for the case $\alpha=1 / 2$. Comparison of radius of univalence of the sections has been shown by providing a table of numerical estimates for the special choices of $\alpha$.
\end{abstract}

Key words: Univalent Harmonic, convex in one direction, closeto-convex, partial sums, sections

2010 Mathematical Subject Classification: Primary: 30C45; Secondary: 31A05, 30C55

1. Introduction and motivation. Harmonic mappings are useful in the study of fluid flow problems (see [3]). Furthermore, univalent harmonic functions having special geometric properties such as convexity, starlikeness, and close-to-convexity arise naturally while dealing with planar fluid dynamics problems. For example, in [3, Theorem 4.5], Aleman et al. considered a fluid flow problem on a convex domain $\Omega_{0}$ satisfying an interesting geometric property. Furthermore, harmonic mappings which appear as solutions to some of these real-world problems are very complicated and, hence, evaluating values of such functions is challenging. In this connection, it is interesting to consider the problem of approximating harmonic mappings by harmonic polynomials without compromising the univalency of the polynomial. With this brief motivation, we shall begin to consider the partial sums for the univalent analytic functions and univalent harmonic mappings.

(c) Petrozavodsk State University, 2018 
Let $\mathcal{S}$ denote the class of all normalized univalent analytic functions $\phi$ defined in the unit disk $\mathbb{D}=\{z:|z|<1\}$ such that

$$
\phi(z)=z+\sum_{k=2}^{\infty} a_{k} z^{k}
$$

For $n \geqslant 2$, the $n^{\text {th }}$ partial sum of $\phi$ is the polynomial defined by

$$
s_{n}(\phi)(z)=z+\sum_{k=2}^{n} a_{k} z^{k} .
$$

In [23] Szegö proved that the partial sum $s_{n}(\phi)$ is univalent in $|z|<1 / 4$ for all $\phi \in \mathcal{S}$ and $n \geqslant 2$. In [19], Robertson proved that the $n^{\text {th }}$ partial sum of the Koebe function is starlike in the disk $|z|<1-3 n^{-1} \log n$, for $n \geqslant 5$, and that 3 cannot be replaced by a smaller constant. The general theorems on convolutions [20] (see also [8, p.256, p. 273]) allow to infer that $s_{n}(\phi)$ is convex, starlike, or close-to-convex in the disk $|z|<$ $<1-3 n^{-1} \log n$, for $n \geqslant 5$, whenever $\phi$ is convex, starlike, or close-toconvex in $\mathbb{D}$ (for details on the class $\mathcal{S}$ and its geometric subclasses, one can refer [8]), respectively. However, the exact radius of univalence $r_{n}$ of $s_{n}(\phi)$ remains an open problem, if $\phi \in \mathcal{S}$. Jenkins [11] proved that $s_{n}(\phi)$ is univalent in $|z|<r_{n}$ for $\phi \in \mathcal{S}$, where the radius of univalence $r_{n}$ is at least $1-(4 \log n-\log (4 \log n)) / n$ for $n \geqslant 8$. However, by making use of the exact coefficient bounds, one could get $r_{n}>1-(4 \log n-2 \log (\log n)) / n$ for $n \geqslant 7$. Moreover, $1-(4 \log n-2 \log (\log n)) / n>1-(4 \log n-\log (4 \log n)) / n$ for $n \geqslant 55$.

Denote by $\mathcal{H}$ the class of all complex-valued harmonic functions $f=h+\bar{g}$ in $\mathbb{D}$ normalized by $h(0)=g(0)=0=h^{\prime}(0)-1$. We call $h$ and $g$ the analytic and the co-analytic parts of $f$, respectively, and, obviously, they have the following power series representation:

$$
h(z)=z+\sum_{k=2}^{\infty} a_{k} z^{k} \text { and } g(z)=\sum_{k=1}^{\infty} b_{k} z^{k}, z \in \mathbb{D} .
$$

We shall use this representation throughout the discussion. The Jacobian $J_{f}$ of $f=h+\bar{g}$ is $J_{f}(z)=\left|h^{\prime}(z)\right|^{2}-\left|g^{\prime}(z)\right|^{2}$. We say that $f$ is sensepreserving in $\mathbb{D}$ if $J_{f}(z)>0$ in $\mathbb{D}$. Let $\mathcal{S}_{H}$ denote the class of all sensepreserving harmonic univalent mappings $f \in \mathcal{H}$ and set $\mathcal{S}_{H}^{0}=\left\{f \in \mathcal{S}_{H}\right.$ : $\left.f_{\bar{z}}(0)=0\right\}$. For many basic results on univalent harmonic mappings in $\mathcal{S}_{H}^{0}$ 
and its well-known geometric subclasses, namely, $\mathcal{K}_{H}^{0}, \mathcal{S}_{H}^{* 0}$, and $\mathcal{C}_{H}^{0}$ that map $\mathbb{D}$ onto convex, starlike, and close-to-convex domains, respectively, we refer to $[7,15]$. Here we recall a new subclass of $\mathcal{S}_{H}^{0}$, namely, $\mathcal{S}_{H}^{0}(\mathcal{S})$ introduced in [16], where

$$
\mathcal{S}_{H}^{0}(\mathcal{S})=\left\{h+\bar{g} \in \mathcal{S}_{H}^{0}: h+e^{i \theta} g \in \mathcal{S} \text { for some } \theta \in \mathbb{R}\right\} .
$$

This class along with a related conjecture concerning convex harmonic mappings were proposed in [16]; this conjecture was later disproved by Kayumov et al in [12]. For $f=h+\bar{g} \in \mathcal{S}_{H}^{0}$ with the power series representation as in (2), the sections/partial sums $s_{n, m}(f)$ of $f$ are defined as

$$
s_{n, m}(f)(z)=s_{n}(h)(z)+\overline{s_{m}(g)(z)},
$$

where $n \geqslant 1$ and $m \geqslant 2$. However, the special case $m=n \geqslant 2$ seems interesting in its own merit. From the definition above it is clear that partial sums of $f$ can be thought of as an approximation of $f$ by complex-valued harmonic polynomials and, thus, approximation of univalent harmonic mappings by univalent harmonic polynomials might lead to new applications. For fundamental results on the partial sums of univalent harmonic mappings, one can refer to $[13,14,17,18]$. The coefficient problem for $\mathcal{S}_{H}^{0}$ remains open. For large values of $n$, coefficient estimates are given in [18]. On the other hand, in a recent paper [1] Ali et al. have shown that if $f=h+\bar{g} \in \mathcal{S}_{H}^{0}$, then $\left|a_{2}\right| \leqslant 20.92$ which is the best known estimation. We recall a few results from [17] which are motivation for the problem which we consider in this article.

Theorem A. Let $f=h+\bar{g} \in \mathcal{S}_{H}^{0}$ with the series representation as in (2). Suppose that $f$ belongs to $\mathcal{C}_{H}^{0}$, the class of close-to-convex harmonic mappings or $\mathcal{S}_{H}^{0}(\mathcal{S})$. Then the section $s_{n, m}(f)$ is univalent in the disk $|z|<r_{n, m}$. Here $r_{n, m}$ is the unique positive root of the equation $\psi(n, m, r)=0$, where

$$
\psi(n, m, r)=\frac{1}{12 r}\left(\frac{1-r}{1+r}\right)^{3}\left[1-\left(\frac{1-r}{1+r}\right)^{6}\right]-R_{n}-T_{m}
$$

with

$$
R_{n}=\sum_{k=n+1}^{\infty} A_{k} r^{k-1}, \quad T_{m}=-\sum_{k=m+1}^{\infty} A_{-k} r^{k-1}
$$

where

$$
A_{k}=\frac{k(k+1)(2 k+1)}{6}
$$


In particular, every section $s_{n, n}(f)(z)$ is univalent in the disk $|z|<r_{n, n}$, where

$$
r_{n, n}>r_{n, n}^{L}:=1-\frac{(7 \log n-4 \log (\log n))}{n} \text { for } n \geqslant 15
$$

Moreover, $r_{n, m} \geqslant r_{l, l}^{L}$, where $l=\min \{n, m\} \geqslant 15$.

We have the following interesting result for functions in the convex family $\mathcal{K}_{H}^{0}$ of harmonic mappings: the lower bound of $r_{n, m}$ can be improved compared to that of the bounds in Theorem A.

Theorem B. Let $f=h+\bar{g} \in \mathcal{K}_{H}^{0}$ with the series representation as in (2). Then the section $s_{n, m}(f)$ is univalent in the disk $|z|<r_{n, m}$, where $r_{n, m}$ is the unique positive root of the equation $\mu(n, m, r)=0$. Here

$$
\mu(n, m, r)=\frac{1-r}{(1+r)^{3}}-\sum_{k=n+1}^{\infty}\left[\frac{k(k+1)}{2} r^{k-1}\right]-\sum_{k=m+1}^{\infty}\left[\frac{k(k-1)}{2} r^{k-1}\right] .
$$

In particular, for $n \geqslant 5$, and $\theta \in \mathbb{R}$, the harmonic function

$$
s_{n, n}(f ; \theta)(z)=s_{n}(h)(z)+e^{i \theta} \overline{s_{n}(g)(z)}
$$

is univalent and close-to-convex in the disk $|z|<1-3 n^{-1} \log n$. Moreover, we have $r_{n, m} \geqslant 1-(4 \log l-2 \log (\log l)) / l$, where $l=\min \{n, m\} \geqslant 7$.

$$
\begin{aligned}
& \text { For }-1 / 2 \leqslant \alpha<1, \text { set } \\
& \mathcal{F}(\alpha)=\left\{h+\bar{g} \in \mathcal{H}: \operatorname{Re}\left(1+\frac{z h^{\prime \prime}(z)}{h^{\prime}(z)}\right)>\alpha, \frac{g^{\prime}(z)}{h^{\prime}(z)}=e^{i \theta} z, \forall z \in \mathbb{D}\right\}
\end{aligned}
$$

and $\mathcal{F}=\mathcal{F}(-1 / 2)$. Note that for $-1 / 2 \leqslant \alpha<1, f \in \mathcal{F}(\alpha)$ has the following integral representation (see [2]):

$$
f(z)=\int_{0}^{z} \int_{|x|=1} \frac{1}{(1-x t)^{2(1-\alpha)}} \mathrm{d} \mu(x) \mathrm{d} t+\int_{0}^{z} \int_{|x|=1} \frac{e^{i \theta} t}{(1-x t)^{2(1-\alpha)}} \mathrm{d} \mu(x) \mathrm{d} t,
$$

for some probability measure $\mu$ defined on $\partial \mathbb{D}:=\{z \in \mathbb{C}:|z|=1\}$.

Remark 1. Let us consider the family of close-to-convex harmonic functions $\mathcal{F}$. A keen observation of the proof of Theorem $B$ reveals that the results in Theorem $B$ are valid for the class $\mathcal{F}$ too (note that members 
of $\mathcal{F}$ are not necessarily even starlike). Comparison of this fact with Theorem A motivates us to consider certain subclasses of close-to-convex harmonic mappings with special condition on the analytic part of $f$.

In this article, we determine the radius of univalence $r_{n, m}$ of $s_{n, m}(f)$, when $f \in \mathcal{F}(\alpha)$, where $\alpha \in[-1 / 2,1)$. The lower bound for $r_{n, m}$ is determined for the special choice of $\alpha$. The paper is organized as follows. In Section 2, we recall a few known results from the literature, which are helpful in the proof of our main theorems. We present our main theorems in Section 3.

2. Useful results. The following result by Bazilevich [5] gives the necessary and sufficient condition for a normalized analytic function to be univalent in $\mathbb{D}$.

Theorem C. An analytic function $\phi$ defined in $\mathbb{D}$ and determined by is univalent in $\mathbb{D}$ if and only if for each $z \in \mathbb{D}$ and each $t \in[0, \pi / 2]$,

$$
\frac{\phi\left(r e^{i \eta}\right)-\phi\left(r e^{i \psi}\right)}{r e^{i \eta}-r e^{i \psi}}:=\sum_{k=1}^{\infty} a_{k} \frac{\sin k t}{\sin t} z^{k-1} \neq 0
$$

where $t=(\eta-\psi) / 2, z=r e^{i(\eta+\psi) / 2}$ and $\left.\frac{\sin k t}{\sin t}\right|_{t=0}=k$.

In [21], Starkov generalized this result to the class of normalized sensepreserving harmonic mappings in the following form.

Theorem D. A sense-preserving harmonic function $f=h+\bar{g}$ defined in $\mathbb{D}$ determined by (2) is univalent in $\mathbb{D}$ if and only if for each $z \in \mathbb{D} \backslash\{0\}$ and each $t \in(0, \pi / 2]$,

$$
\frac{f\left(r e^{i \eta}\right)-f\left(r e^{i \psi}\right)}{r e^{i \eta}-r e^{i \psi}}:=\sum_{k=1}^{\infty}\left[\left(a_{k} z^{k}-\overline{b_{k} z^{k}}\right) \frac{\sin k t}{\sin t}\right] \neq 0,
$$

where $t=(\eta-\psi) / 2$ and $z=r e^{i(\eta+\psi) / 2}$.

Further extension of Theorem D along with its use may be obtained from the article of Amozova et al. [4]. The following two-points distortion theorem plays a crucial role in the proof of our main results.

Theorem E. If $f \in \mathcal{S}, r \in(0,1), t, \psi \in \mathbb{R}$ such that $t \neq \psi$, then

$$
\left|\frac{f\left(r e^{i t}\right)-f\left(r e^{i \psi}\right)}{r e^{i t}-r e^{i \psi}}\right| \geqslant \frac{1-r^{2}}{r^{2}}\left|f\left(r e^{i t}\right)\right| \cdot\left|f\left(r e^{i \psi}\right)\right| .
$$


3. The distortion theorem and the partial sums problem. By making use of Theorem E, we derive a two-points distortion theorem for functions in the family $\mathcal{F}(\alpha)$. This result is crucial in the proof of our main theorem.

Theorem 1. (Two points distortion theorem) Suppose that $f=h+\bar{g} \in \mathcal{F}(\alpha)$ for some $\alpha \in[-1 / 2,1)$. For each $\lambda \in \mathbb{C}$ such that $|\lambda|=1$, define

$$
F_{\lambda}(z):=h(z)+\lambda g(z) .
$$

Then for $0<r<1$ and $t, \psi \in \mathbb{R}$ such that $t \neq \psi, f$ satisfies the following inequalities

$$
\begin{aligned}
\left|\frac{F_{\lambda}\left(r e^{i t}\right)-F_{\lambda}\left(r e^{i \psi}\right)}{r e^{i t}-r e^{i \psi}}\right| & \geqslant \frac{1-r^{2}}{r^{2}} L^{2}(r, \alpha)=: A(r, \alpha), \\
\left|\frac{f\left(r e^{i t}\right)-f\left(r e^{i \psi}\right)}{r e^{i t}-r e^{i \psi}}\right| & \geqslant A(r, \alpha) \text { for } 0<r<1,
\end{aligned}
$$

where

$$
L(r, \alpha):= \begin{cases}\frac{2 r}{1+r}-\log (1+r) & \text { if } \alpha=0, \\ 2 \log (1+r)-r & \text { if } \alpha=1 / 2, \\ \frac{(1+r+2 \alpha(1-r))(1+r)^{2 \alpha-1}-(1+2 \alpha)}{2 \alpha(2 \alpha-1)} & \\ & \text { if } \alpha \in[-1 / 2,1) \backslash\{0,1 / 2\} .\end{cases}
$$

Proof. Let $f=h+\bar{g} \in \mathcal{F}(\alpha)$ for some $\alpha \in[-1 / 2,1)$ and $F_{\lambda}(z):=h(z)+\lambda g(z)$. Set $f_{\lambda}=h+\lambda \bar{g}$. It is clear that both $F_{\lambda}$ and $f_{\lambda}$ are univalent and close-to-convex in $\mathbb{D}$ for all $\lambda$ such that $|\lambda|=1$, which follow from a result of Bharanedhar et al. [6]. For every pair of points $z_{1}=r e^{i t}$ and $z_{2}=r e^{i \psi}$, we can find a $\lambda$ such that

$$
\left(h\left(z_{1}\right)-h\left(z_{2}\right)\right)+\overline{\left(g\left(z_{1}\right)-g\left(z_{2}\right)\right)}=\left(h\left(z_{1}\right)-h\left(z_{2}\right)\right)+\lambda\left(g\left(z_{1}\right)-g\left(z_{2}\right)\right) .
$$

Therefore, by using Theorem E, we get

$$
\begin{aligned}
\left|\frac{f\left(r e^{i t}\right)-f\left(r e^{i \psi}\right)}{r e^{i t}-r e^{i \psi}}\right| & =\left|\frac{F_{\lambda}\left(r e^{i t}\right)-F_{\lambda}\left(r e^{i \psi}\right)}{r e^{i t}-r e^{i \psi}}\right| \geqslant \\
& \geqslant \frac{1-r^{2}}{r^{2}}\left|F_{\lambda}\left(r e^{i t}\right)\right| \cdot\left|F_{\lambda}\left(r e^{i \psi}\right)\right| .
\end{aligned}
$$


In order to complete the proof, we need to find the lower bounds of $\left|F_{\lambda}(z)\right|$.

Let $\gamma$ be the preimage under $F_{\lambda}$ of the line segment joining 0 and $F_{\lambda}(z)$. Then

$$
\begin{gathered}
\left|F_{\lambda}(z)\right|=\int_{\gamma}\left|F_{\lambda}^{\prime}(\zeta)\right| \cdot|d \zeta| \geqslant \int_{\gamma}\left(\left|h^{\prime}(\zeta)\right|-\left|g^{\prime}(\zeta)\right|\right) \cdot|d \zeta| \geqslant \\
\geqslant \int_{\gamma}(1-|\zeta|) \cdot\left|h^{\prime}(\zeta)\right| \cdot|d \zeta| \geqslant \int_{0}^{r} \frac{(1-\rho)}{(1+\rho)^{2(1-\alpha)}} d \rho,
\end{gathered}
$$

where the last inequality is a consequence of the following well known distortion theorem for the convex function of order $\alpha$ (see [9, p. 139, Vol. 1, Theorem 1] for the case $\alpha$ such that $0 \leqslant \alpha<1)$ )

$$
\left|h^{\prime}(z)\right| \geqslant \frac{1}{(1+r)^{2(1-\alpha)}}, r=|z|<1 .
$$

Also, for $\alpha \in[-1 / 2,0)$, the above inequality holds true, which can be obtained using the same proof technique. The desired conclusion follows, if we use the inequality (8) in (7).

Next, we provide the sharp coefficient estimates for the functions in the family $\mathcal{F}(\alpha)$.

Theorem 2. Suppose that $f=h+\bar{g} \in \mathcal{F}(\alpha)$ for some $\alpha \in[-1 / 2,1)$ with the series representation as in (2). Then, for all $n \geqslant 2$, the coefficients of $f$ satisfy the following inequalities:

$$
\left|a_{n}\right| \leqslant A_{n}(\alpha) \text { and }\left|b_{n}\right| \leqslant \frac{n-1}{n-2 \alpha} A_{n}(\alpha)
$$

where

$$
A_{n}(\alpha)=\frac{1}{n !} \prod_{j=2}^{n}(j-2 \alpha) .
$$

All these bounds are sharp and each inequality becomes equality for the close-to-convex functions $f_{\alpha}(z)=h_{\alpha}(z)+\overline{g_{\alpha}(z)}$ and their rotations, where

$$
f_{\alpha}(z)=\int_{0}^{z} \frac{1}{(1-t)^{2(1-\alpha)}} d t+\overline{e^{i \theta} \int_{0}^{z} \frac{t}{(1-t)^{2(1-\alpha)}} d t}
$$


Proof. The proof follows from the coefficient bounds for the convex functions of order $\alpha$ (see [9, p. 140, Vol. 1, Theorem 2]) and a result of T. J. Suffridge [22, Theorem 5.6]. We remark that the original proofs given in the above references are considered for the values of $\alpha \in[0,1)$, which can be extended to the case of $\alpha \in[-1 / 2,0)$; we skip the details here as the computations are similar (See also [24, Lemma 3.1, Theorem $3.2])$.

Theorem 3. Let $f=h+\bar{g} \in \mathcal{F}(\alpha)$, for some $\alpha \in[-1 / 2,1)$ with the series representation as in (2). Then, for $\theta \in \mathbb{R}$, the harmonic function

$$
s_{n, m}(f ; \theta)(z)=s_{n}(h)(z)+e^{i \theta} \overline{s_{m}(g)(z)}
$$

is univalent in the disk $|z|<r_{n, m}$, where $r_{n, m}$ is the unique positive root of the equation $\psi(n, m, r, \alpha)=0$ in $(0,1)$. Here

$$
\psi(n, m, r, \alpha)=A(r, \alpha)-\sum_{k=n+1}^{\infty}\left[k A_{k}(\alpha) r^{k-1}\right]-\sum_{k=m+1}^{\infty}\left[\frac{k(k-1)}{(k-2 \alpha)} A_{k}(\alpha) r^{k-1}\right],
$$

where $A(r, \alpha)$ and $A_{k}(\alpha)$ are defined as in (6) and (10), respectively. When $\alpha=1 / 2$, we have $r_{n, m} \geqslant 1-(2 \log l) / l$, where $l=\min \{n, m\} \geqslant 3$.

Proof. Suppose that $f=h+\bar{g} \in \mathcal{F}(\alpha)$ for some $\alpha \in[-1 / 2,1)$ with the series representation as in (2). For $0<r<1$ and $\theta \in \mathbb{R}$, we set

$$
F_{r, \theta}(z)=\frac{h(r z)}{z}+e^{i \theta} \frac{g(r z)}{z}
$$

and one could see that

$$
F_{r, \theta}(z)=z+\sum_{k=2}^{\infty} a_{k} r^{k-1} z^{k}+e^{i \theta} \sum_{k=2}^{\infty} b_{k} r^{k-1} z^{k}
$$

It is clear, from the definition of $F_{r, \theta}$, that finding the largest radius of univalence of $s_{n, m}(f ; \theta)$ for every value of $\theta \in[0,2 \pi]$ is equivalent to finding the largest value $r$ such that $s_{n, m}\left(F_{r, \theta}\right)$ is univalent in $\mathbb{D}$ for every $\theta \in[0,2 \pi]$ (See [10] for the details). Using Theorem C, we see that $s_{n, m}\left(F_{r, \theta}\right)$ is univalent in $\mathbb{D}$ if and only if the associated section $P_{n, m, r, \alpha}(z)$ has the property that

$$
P_{n, m, r, \alpha}(z):=\sum_{k=1}^{M}\left[\left(a_{k}^{\prime} z^{k}+b_{k}^{\prime} z^{k}\right) \frac{\sin k t}{\sin t}\right] \neq 0, \text { for all } z \in \mathbb{D} \backslash\{0\},
$$


and $t \in[0, \pi / 2]$, where $M=\max \{n, m\}, l=\min \{n, m\}, a_{k}^{\prime}=a_{k} r^{k-1}$, $b_{k}^{\prime}=b_{k} r^{k-1}$ for all $k \leqslant l$,

$$
\begin{gathered}
a_{k}^{\prime}= \begin{cases}a_{k} r^{k-1} & \text { for all } k>l \text { if } M=n, \\
0 & \text { for all } k>l \text { if } M>n,\end{cases} \\
b_{k}^{\prime}= \begin{cases}e^{i \theta} b_{k} r^{k-1} & \text { for all } k>l \text { if } M=m, \\
0 & \text { for all } k>l \text { if } M>m .\end{cases}
\end{gathered}
$$

Setting $t=(\eta-\psi) / 2, z=\rho e^{i(\eta+\psi) / 2} \in \mathbb{D}$ in (5) and using univalency of $F_{r, \theta}$ for $0<r<1$, we get

$$
\left|\sum_{k=1}^{\infty}\left[\left(a_{k} z^{k}+e^{i \theta} b_{k} z^{k}\right) r^{k-1} \frac{\sin k t}{\sin t}\right]\right| \geqslant A(r, \alpha),
$$

where $A(r, \alpha)$ is defined as in (6). In order to find a lower bound for $\left|P_{n, m, r, \alpha}(z)\right|$, we need to find an upper bound for

$$
\left|R_{n, m, r, \alpha}(z)\right|=\left|\sum_{k=n+1}^{\infty}\left[a_{k} r^{k-1} z^{k} \frac{\sin k t}{\sin t}\right]+\sum_{k=m+1}^{\infty}\left[e^{i \theta}\left(b_{k} r^{k-1} z^{k}\right) \frac{\sin k t}{\sin t}\right]\right|
$$

From Theorem 2, it follows that

$$
\begin{aligned}
\left|R_{n, m, r, \alpha}(z)\right| & \leqslant \sum_{k=n+1}^{\infty}\left[k A_{k}(\alpha) r^{k-1}\right]+\sum_{k=m+1}^{\infty}\left[\frac{k(k-1)}{(k-2 \alpha)} A_{k}(\alpha) r^{k-1}\right]= \\
& =R_{n}(r, \alpha)+T_{m}(r, \alpha)
\end{aligned}
$$

where $A_{k}(\alpha)$ is defined as in (9). From (13) and (14), we get

$$
\left|P_{n, m, r, \alpha}(z)\right| \geqslant A(r, \alpha)-R_{n}(r, \alpha)-T_{m}(r, \alpha)=\psi(n, m, r, \alpha) .
$$

The quantity $P_{n, m, r, \alpha}(z) \neq 0$ holds for all $z \in \mathbb{D} \backslash\{0\}$, whenever $\psi(n, m, r, \alpha)$ defined by (12) is positive. This gives $\psi(n, m, r, \alpha)>0$ for all $r \in\left(0, r_{n, m}\right)$, where $r_{n, m}$ is the positive root of the equation $\psi(n, m, r, \alpha)=0$, which lies in the interval $(0,1)$. This observation proves that $F_{r, \theta}$ is univalent in $\mathbb{D}$ for all $\theta \in[0,2 \pi]$; this implies univalency of $s_{n, m}(f ; \theta)$ (see [10]) in the disk $|z|<r_{n, m}$ for all $\theta \in[0,2 \pi]$. This completes the proof of the first part of the theorem.

From the above discussion, it is clear that $r_{n, m} \geqslant r_{l, l}$, where $l=\min \{n, m\} \geqslant 2$. Next, we shall consider the special case $m=n$ 
and determine the lower bound for $r_{n, n}$ with a certain restriction on $n$ and special choices of $\alpha$.

Let us consider the case $\alpha=1 / 2$. In this case, the sufficient condition (12) for the univalence of $s_{n, n}(f ; \theta)$ reduces to

$$
\begin{gathered}
\psi(n, n, r, 1 / 2)=A(r, 1 / 2)-R_{n}(r, 1 / 2)-T_{n}(r, 1 / 2)= \\
=\left(1-r^{2}\right)\left(\frac{2 \log (1+r)-r}{r}\right)^{2}-2 \sum_{k=n+1}^{\infty} r^{k-1} \geqslant \frac{1-r}{(1+r)^{2}}-\frac{2 r^{n}}{(1-r)} .
\end{gathered}
$$

From the fact that $\lim _{n \rightarrow \infty} \psi(n, n, r, 1 / 2)>0$ for all $r$ such that $0<r<1$, it is clear that the radius of univalence $r_{n, n} \rightarrow 1$ as $n \rightarrow \infty$. Setting $r=1-x / n$, where $x=o(n)$, we see that

$$
\psi(n, n, r, 1 / 2)=\frac{(1-r)^{2}-2(1+r)^{2} r^{n}}{(1-r)(1+r)^{2}}>0,
$$

whenever $0<t(x, n)<1$, where

$$
t(x, n)=2 e^{-x}\left(\frac{2 n}{x}-1\right)^{2} .
$$

Now, we shall prove that $r_{n, n}>1-\gamma_{n} / n$ and we shall explicitly determine the value $\gamma_{n}$ for large values of $n$. In order to prove that, it is sufficient to prove that $t(x, n)$ is a decreasing function in $x$, whenever

$$
\gamma_{n} \leqslant x \leqslant n, \quad 0<t\left(\gamma_{n}, n\right)<1 \text { and } t(n, n)>0 .
$$

Since $e^{x} t(x, n)=O\left(n^{2}\right)$, we may set $\gamma_{n}=2 \log n$. It is easy to see that $1-\gamma_{n} / n>0$ and increases for all $n \geqslant 3$. For $n \geqslant 3$, we shall prove that $r_{n, n}>1-\gamma_{n} / n$.

Fix an integer $n \geqslant 3$ and consider an $x \in\left[\gamma_{n}, n\right]$; we prove that $t(x, n)$ is a decreasing function with respect to $x$. From the definition of $t(x, n)$, it is clear that it is a product of two positive decreasing functions. Hence, $t(x, n)$ is a decreasing function with respect to $x$ for every fixed integer $n \geqslant 3$. Further, it is easy to see that $t(n, n)<1$ for all $n \geqslant 3$. In order to complete the proof, it is enough to show that $t\left(\gamma_{n}, n\right)<1$ for all $n \geqslant 3$. Note that $t\left(\gamma_{n}, n\right)=\frac{2}{n^{2}}\left(\frac{n}{\log n}-1\right)^{2}=2\left(\frac{1}{\log n}-\frac{1}{n}\right)^{2}<1$ for all $n \geqslant 3$. The proof is complete.

Remark 2. From Theorem $B$, we could see that whenever $f \in \mathcal{F}(-1 / 2)$, $s_{n, m}(f ; \theta)$ is univalent in the disk $|z|<r_{n, m}$, where

$$
r_{n, m}>r_{l, l}=1-(4 \log l-2 \log (\log l)) / l
$$


for $l=\min \{m, n\} \geqslant 7$.

Generally, it is possible to estimate lower bounds for $r_{n, m}$ for functions $f \in \mathcal{F}(\alpha)$ for $\alpha \in[-1 / 2,1)$; however, if we consider the computation for finding the lower bound on $r_{n, m}$ in Theorem 3 and Theorem $\mathrm{B}$, the difficulty in handling the general case $\alpha \in[-1 / 2,1)$ becomes clear. Nevertheless, we can compare the variations on the radius of univalence of the partial sums, when we consider functions $f \in \mathcal{F}(\alpha)$ for various values of $\alpha \in[-1 / 2,1)$. We demonstrate this in the following Corollary.

Corollary. Suppose that $f \in \mathcal{F}(\alpha)$ for some $\alpha \in[-1 / 2,1)$. Then the value of $n$ for which $s_{n, n}(f ; \theta)$ is univalent in the disk $|z|<\rho$ for all values of $\theta \in[0,2 \pi]$ is given in Table 1 :

\begin{tabular}{|l|c|c|c|c|c|c|}
\hline$|z|<\rho$ & $\alpha=-\frac{1}{2}$ & $\alpha=-\frac{\mathbf{1}}{\mathbf{4}}$ & $\alpha=\mathbf{0}$ & $\alpha=\frac{\mathbf{1}}{\mathbf{4}}$ & $\alpha=\frac{\mathbf{1}}{\mathbf{2}}$ & $\alpha=\frac{\mathbf{3}}{\mathbf{4}}$ \\
\hline $\mathbf{1 / 4}$ & $n \geqslant 2$ & $n \geqslant 2$ & $n \geqslant 2$ & $n \geqslant 2$ & $n \geqslant 2$ & $n \geqslant 2$ \\
\hline $\mathbf{1} / \mathbf{2}$ & $n \geqslant 12$ & $n \geqslant 10$ & $n \geqslant 8$ & $n \geqslant 6$ & $n \geqslant 4$ & $n \geqslant 3$ \\
\hline $\mathbf{3 / 4}$ & $n \geqslant 43$ & $n \geqslant 36$ & $n \geqslant 29$ & $n \geqslant 22$ & $n \geqslant 16$ & $n \geqslant 9$ \\
\hline $\mathbf{9} / \mathbf{1 0}$ & $n \geqslant 160$ & $n \geqslant 135$ & $n \geqslant 111$ & $n \geqslant 86$ & $n \geqslant 61$ & $n \geqslant 35$ \\
\hline
\end{tabular}

Table 1: Values of $n$ for which $s_{n, n}(f ; \theta)$ is univalent in the disk $|z|<\rho$

Proof. The lower bound on $\rho$, corresponding to the value $n=2$, follows from the result [10, Proposition 2.1] and the result of Szegö [23]. All the other lower bounds have been estimated with the help of the Mathematica software by estimating $\psi(n, n, r, \alpha)=0$ in the equation (12), corresponding to the values of $r=\rho$, fixing $\alpha=-1 / 2,-1 / 4,0,1 / 4,1 / 2,3 / 4$.

Acknowledgment. The author thanks Prof. S. Ponnusamy for fruitful discussion and comments on this manuscript. The author also thanks the referees for their comments and suggestions, which helped the author to improve the results in this article.

\section{References}

[1] Y. Abu Muhanna, R. M. Ali, and S. Ponnusamy, The spherical metric and univalent harmonic mappings, Monatsh. Math., 2018, 14 pages; Available online. DOI: https://doi.org/10.1007/s00605-018-1160-4. 
[2] Y. Abu Muhanna, L. Li, and S. Ponnusamy, Extremal problems on the class of convex functions of order $-1 / 2$, Arch. Math., 2014, vol. 103, pp. 461-471. DOI: https://doi.org/10.1007/s00013-014-0705-6.

[3] A. Aleman, and A. Constantin, Harmonic maps and ideal fluid flows, Arch. Rational Mech. Anal., 2014, vol. 204, pp. 479-513. DOI: https://doi. org/10.1007/s00205-011-0483-2.

[4] K. F. Amozova, E. G. Ganenkova, and S. Ponnusamy, Criteria of univalence and fully $\alpha$-accessibility for $p$-harmonic and p-analytic functions, Complex Var. Elliptic Equ., 2017, vol. 62, no. 8, pp. 1165-1183. DOI: https://doi.org/10.1080/17476933.2016.1273908.

[5] I. E. Bazilevich, The problem of coefficients of univalent functions, Math. J. of the Aviation Institute (Moscow), 1945, pp. 29-47.

[6] S. V. Bharanedhar, and S. Ponnusamy, Coefficient conditions for harmonic univalent mappings and hypergeometric mappings, Rocky Mountain J. Math., 2014 vol. 44, no. 3, pp. 753-777. DOI: https://doi.org/10. 1216/RMJ-2014-44-3-753.

[7] P. Duren, Harmonic mappings in the plane, Cambridge Tracts in Mathematics, 156, Cambridge Univ. Press, Cambridge, 2004.

[8] P. Duren, Univalent functions (Grundlehren der mathematischen Wissenschaften 259, New York, Berlin, Heidelberg, Tokyo), SpringerVerlag, 1983.

[9] A. W. Goodman, Univalent functions, Vols. 1-2, Mariner, Tampa, Florida, 1983.

[10] R. Hernández, and M. J. Martín, Stable geometric properties of analytic and harmonic functions, Math. Proc. Camb. Phil. Soc. 2013, vol. 155, no. 2, pp. 343-359. DOI: https://doi.org/10.1017/S0305004113000340.

[11] J. A. Jenkins, On an inequality of Golusin, Amer. J. Math., 1951, vol. 73, pp. 181-185.

[12] I. R. Kayumov, S. Ponnusamy, and L. A. Xuan, Rotations of convex harmonic univalent mappings, Bull. Sci. Math., 2018, 8 pages, To appear.

[13] L. Li, and S. Ponnusamy, Injectivity of sections of univalent harmonic mappings, Nonlinear Analysis, 2013, vol. 89, pp. 276-283. DOI: https: //doi.org/10.1016/j.na.2013.05.016.

[14] L. Li, and S. Ponnusamy, Sections of stable harmonic convex functions, Nonlinear Analysis, 2015, vol. 123-124, pp. 178-190. DOI: https://doi. org/10.1016/j.na.2014.06.005.

[15] S. Ponnusamy and A. Rasila, Planar harmonic and quasiregular mappings, Topics in Modern Function Theory: Chapter in CMFT, RMS-Lecture Notes Series No. 19, 2013, pp. 267-333. 
[16] S. Ponnusamy and A. Sairam Kaliraj, On the coefficient conjecture of Clunie and Sheil-Small on univalent harmonic mappings, Proc. Indian Acad. Sci., 2015, vol. 125, no. 3, pp. 277-290. DOI: https://doi.org/10.1007/ s12044-015-0236-5.

[17] S. Ponnusamy, A. Sairam Kaliraj, and V. V. Starkov, Sections of univalent harmonic mappings, Indagationes Mathematicae, 2017, vol. 28, no. 2, pp. 527-540. DOI: https://doi.org/10.1016/j.indag.2017.01.001.

[18] S. Ponnusamy, A. Sairam Kaliraj, and V. V. Starkov, Coefficients of univalent harmonic mappings, Monatshefte für Mathematik, 2017, Available Online. DOI: https://doi.org/10.1007/s00605-017-1038-x.

[19] M. S. Robertson, The partial sums of multivalently star-like functions, Ann. of Math., 1941, vol. 42, no. 4, pp. 829-838.

[20] St. Ruscheweyh, and T. Sheil-Small, Hadamard products of Schlicht functions and the Pólya-Schoenberg conjecture, Comment. Math. Helv., 1973, vol. 48, no. 1, pp. 119-135.

[21] V. V. Starkov, Univalence of harmonic functions, problem of Ponnusamy and Sairam, and constructions of univalent polynomials, Probl. Anal. Issues Anal., 2014, vol. 3(21), no.2, pp. 59-73. DOI: https://doi.org/10. 15393/j3.art. 2014.2729.

[22] T. J. Suffridge, Some special classes of conformal mappings, in Handbook of complex analysis: Geometric function theory, Vol. 2, Elsevier, Amsterdam, 2005.

[23] G. Szegö, Zur Theorie der schlichten Abbildungen, Math. Ann., 1928, vol. 100 , no. 1 , pp. 188-211.

[24] Z. G. Wang, Z. H. Liu, A. Rasila, and Y. Sun, On a problem of Bharanedhar and Ponnusamy involving planar harmonic mappings, Rocky Mountain J. Math., 2018, vol. 48, no. 4, pp. 1345-1358. DOI: https://doi.org/10. 1216/RMJ-2018-48-4-1345.

Received February 24, 2018.

In revised form, November 28, 2018.

Accepted November 28, 2018.

Published online December 13, 2018.

Indian Institute of Technology Ropar,

Nangal Road, Rupnagar, Punjab, 140001, India.

Email: sairamkaliraj@gmail.com; sairam@iitrpr.ac.in 